\title{
Técnica híbrida (TH) versus estándar (TS) en la punción con aguja fina guiada por ecoendoscopia para lesiones sólidas del páncreas
}

\section{Hybrid technique versus standard technique for endoscopic ultrasound guided fine needle aspiration of solid pancreatic lesions}

\author{
Martín Alonso Gómez*, Oscar Ruíz², Adán Lúquez Mendiola³
}

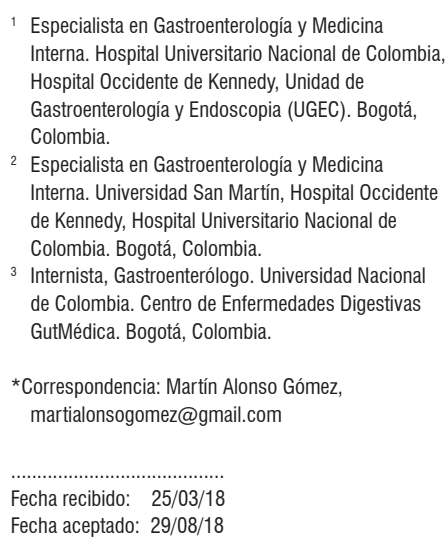

2 Especialista en Gastroenterología y Medicina Interna. Universidad San Martín, Hospital Occidente de Kennedy, Hospital Universitario Nacional de Colombia. Bogotá, Colombia.

${ }_{3}$ Internista, Gastroenterólogo. Universidad Nacional de Colombia. Centro de Enfermedades Digestivas GutMédica. Bogotá, Colombia.

*Correspondencia: Martín Alonso Gómez, martialonsogomez@gmail.com

Fecha recibido: $\quad 25 / 03 / 18$ Fecha aceptado: 29/08/18

\begin{abstract}
Resumen
La ultrasonografía endoscópica (USE) se usa ampliamente para evaluar enfermedades pancreatobiliares, especialmente masas pancreáticas. La USE tiene una buena capacidad para detectar masas pancreáticas, pero no es suficiente para el diagnóstico diferencial de varios tipos de lesiones. La aspiración endoscópica con aguja fina guiada por ultrasonido (USE-PAF) es el método de diagnóstico de elección para masas pancreáticas y su precisión se afecta por diversos métodos de punción. Materiales y métodos: nuestro objetivo fue evaluar el rendimiento diagnóstico de la técnica de succión estándar (TS) versus la técnica húmeda híbrida $(\mathrm{TH})$ en el estudio de lesiones sólidas en páncreas, utilizando un diseño prospectivo, con ocultación única, aleatorizado y controlado, que incluye a pacientes con diagnóstico de lesión sólida en páncreas a los que se realizó USE-PAF desde mayo de 2014 a junio de 2016. Resultados: en total incluimos 65 pacientes, $34(52,3 \%)$ se asignaron a USE-PAF con TH y $31(47,7 \%)$ pacientes a USE-PAF con TS. Se encontró que la frecuencia relativa porcentual respecto a la técnica de punción en la USE-PAF en lesiones sólidas de páncreas, que permite obtener la cantidad de tejido adecuado para el diagnóstico citológico, fue de 85,2\% para la TH y $71 \%$ para la TS, con un OR de 2,35 (IC 95\%; 1,2-4,7) a favor de la TH. Conclusión: este estudio sugiere que la TH es superior a la TS en el diagnóstico de las lesiones sólidas del páncreas, por lo cual, dado que la implementación de esta técnica no aumenta costos y es muy sencilla, sugerimos que sea la técnica de elección cuando se necesita puncionar una lesión sólida.
\end{abstract}

Palabras clave

Ultrasonografía endoscópica, cáncer de páncreas, punción aguja fina, citología.

\begin{abstract}
Endoscopic ultrasound (EUS) is widely used to evaluate pancreatobiliary diseases, especially pancreatic masses. EUS has a good ability to detect pancreatic masses, but it is not sufficient for differential diagnoses of various types of lesions. Endoscopic ultrasound-guided fine needle aspiration (EUS-FNA) is the diagnostic method of choice for pancreatic masses, but its accuracy is affected by various puncture methods. Materials and methods: Our objective was to compare the diagnostic yield of examinations of solid lesions in the pancreas by the standard suction technique (ST) with the yield of the hybrid technique (HT) using a prospective, single blind, randomized, controlled design. Patients diagnosed with solid pancreatic lesions who underwent EUS-FNA from May 2014 to June 2016 were included. Results: We included 65 patients, 34 of whom (52.3\%) were assigned to EUS-FNA with HT, and 31 of whom (47.7\%) were assigned to EUS-FNA with TS. We found that the relative frequency that HT successfully obtained an adequate amount of tissue for the cytological diagnosis was $85.2 \%$ while ST's relative frequency of success was $71 \%$. The odds ratio was $2.35(95 \% \mathrm{Cl}$; 1.2-4.7) in favor of HT. Conclusion: This study suggests that the TH is superior to ST for diagnosis of solid pancreatic lesions. Since implementation of this technique does not increase costs and is very simple, we suggest that it become the technique of choice for EUS-FNA.
\end{abstract}

\section{Keywords}

Endoscopic ultrasound, pancreatic cancer, fine needle puncture, cytology. 


\section{INTRODUCCIÓN}

Las lesiones sólidas del páncreas representan un grupo heterogéneo de entidades que pueden ser clasificadas como neoplásicas y no neoplásicas. Las lesiones neoplásicas son las más comunes, estas incluyen adenocarcinoma, tumores neuroendocrinos, tumor pseudopapilar sólido, pancreatoblastoma, linfoma, metástasis y neoplasias misceláneas raras (1). El adenocarcinoma ductal es el tumor maligno más frecuente del páncreas y representa cerca del $90 \%$ de todos las neoplasias malignas pancreáticas (2). Es una causa significativa de mortalidad, la tasa de sobrevida a 5 años es menor del $5 \%$, pero puede alcanzar el $20 \%$ en pacientes seleccionados con tumores no invasivos a quienes se ha realizado resección quirúrgica y su objetivo es detectarlo en estadios tempranos (3). Actualmente, el ultrasonido, la tomografía computarizada y las imágenes por resonancia magnética son el pilar en la evaluación del 80\%-85\% de las lesiones sólidas en páncreas (4). El diagnóstico preoperatorio de las lesiones sólidas pancreáticas es un reto, a pesar del avance tecnológico en las imágenes. La ecoendoscopia más punción con aguja fina es considerado el método de elección para detectar y diagnosticar estas lesiones (5). El rendimiento diagnóstico es altamente sensible y específico; sin embargo, varios factores afectan este rendimiento, dentro de estos se encuentran la experiencia del ecoendoscopista, posición del equipo, momento del día, número de aguja, técnica utilizada, característica de las lesiones, número de pases, citólogo en sala, pancreatitis crónica, entre otros (6-16).

Para el abordaje de las lesiones sólidas en páncreas se han desarrollado dos técnicas principales de succión: técnica seca y técnica húmeda (17). La técnica seca estándar (TS) consiste en los siguientes pasos: visualización por ecoendoscopia de la lesión en una posición óptima, inserción de la aguja (calibre 22), selección de la línea de punción, puncionar con la aguja, remoción del estilete, succión por jeringa de vacío, movimientos de un lado para otro de la aguja, retiro de la aguja y expulsión de la muestra de la aguja utilizando el estilete (18). La técnica húmeda ha sido desarrollada recientemente para mejorar la calidad de las muestras. En esta técnica, antes de puncionar la lesión se retira el estilete (aguja calibre 22) y se prelava con $5 \mathrm{~mL}$ de solución salina para reemplazar la columna de aire con líquido. Una jeringa de $10 \mathrm{~mL}$ se prellena con $3 \mathrm{~mL}$ de solución salina y se utiliza para aspirar luego de realizar la punción de la lesión. Una vez la aguja está dentro de la lesión, esta se mueve 3 veces de un lado para otro, esta maniobra se repite 4 veces (pases), para un total de 12 movimientos. Al retirar la aguja, el aspirado se libera en una lámina y se aplica aire, esto es más eficiente y seguro para sacar el aspirado que reinsertar el estilete $(17,19)$. Un metaanálisis reciente mostró mayor sangrado en el grupo donde el estilete fue reinsertado para sacar el aspirado (20). La técnica híbrida (TH) consiste en realizar los mismos pasos de la técnica húmeda inicial, pero colocar en la aguja una jeringa premontada con vacío, la cual se activa una vez la aguja está dentro de la lesión.

El objetivo del presente trabajo es determinar el rendimiento diagnóstico de la técnica de succión seca estándar versus la técnica de succión húmeda híbrida en el estudio de lesiones sólidas del páncreas en una institución hospitalaria de tercer nivel en Bogotá.

\section{MATERIALES Y MÉTODO}

Este trabajo presenta la experiencia en una institución hospitalaria de tercer nivel en Bogotá. Se utilizó un diseño prospectivo, con ocultación única, aleatorizado y controlado para investigar qué técnica de punción con aguja fina guiada por ecoendoscopia en lesiones sólidas de páncreas permite obtener la cantidad de tejido adecuado para el diagnóstico patológico. Se incluyeron pacientes con diagnóstico de lesión sólida en páncreas, que ingresaron a sala de procedimientos para realización de ecoendoscopia más punción con aguja fina entre mayo de 2014 y junio de 2016. Los criterios de inclusión y exclusión se resumen en la Tabla 1 y Tabla 2. Los procedimientos se realizaron en la sala de gastroenterología de un hospital de tercer nivel en Bogotá, bajo sedación guiada por anestesiólogo, con una combinación de propofol más remifentanilo. Todas las punciones fueron guiadas por ecoendoscopia con un equipo marca Pentax, un endosonógrafo lineal y realizadas por un endoscopista experimentado en procedimientos intervencionistas, con más de 1000 ecoendoscopias con punción realizadas.

Tabla 1. Criterios de inclusión y exclusión

\begin{tabular}{l}
\hline Criterios de inclusión \\
\hline Pacientes con masa pancreática a quienes se hace referencia de \\
punción con aguja fina guiada por ecoendoscopia (USE-PAF) \\
\hline Criterios de exclusión \\
\hline Pacientes con funcionalidad disminuida mayor de 4 en la escala de \\
ECOG (Tabla 2) \\
Pacientes con riesgo de hemorragia (INR $>1,5$ o con recuento de \\
plaquetas $<50000 / \mathrm{mm}^{2}$ ) \\
Pacientes que consumen 2 o más agentes antiagregantes \\
plaquetarios \\
Pacientes con masa pancreática que no se logra detectar por \\
ultrasonografía endoscópica (USE) \\
Mujer embarazada \\
Pacientes menores de 18 años
\end{tabular}

ECOG: Grupo Cooperativo Oncológico del Este; INR: índice internacional normalizado. 
Tabla 2. Escala de calidad de vida del paciente ECOG (21)

\begin{tabular}{cl}
\hline Grado & \\
\hline 0 & Totalmente activo, capaz de llevar a cabo todas las actividades de la vida diaria, previo a la enfermedad sin restricción \\
1 & El paciente presenta síntomas que le impiden realizar trabajos arduos, aunque se desempeña normalmente en sus actividades cotidianas y \\
en trabajos ligeros & El paciente solo permanece en la cama durante las horas de sueño nocturno \\
2 & $\begin{array}{l}\text { El paciente no es capaz de desempeñar ningún trabajo, se encuentra con síntomas que le obligan a permanecer en la cama durante varias } \\
\text { horas al día, además de las de la noche, pero que no superan el } 50 \% \text { del día }\end{array}$ \\
$3 \quad$ El individuo satisface la mayoría de sus necesidades personales solo
\end{tabular}

Se utilizaron agujas de aspiración para ecoendoscopia número 22 gauge (Boston Scientific). Se usó la técnica de succión híbrida y la técnica de succión seca $(10 \mathrm{~mL})$ estándar con estilete para la toma de biopsias, con un total de 3 pases y 4 movimientos dentro de la lesión, según las recomendaciones descritas en la literatura (17-19). Las muestras se extendieron en láminas y se fijaron en alcohol etílico, estas se enviaron para estudio patológico por un especialista en citología del páncreas, el cual no conocía el método TH vs. TS utilizado para obtener las muestras. La información se recopiló en Google Drive y se obtuvieron variables cuantitativas discretas, las cuales se expresaron en frecuencias absolutas y relativas, calculando la frecuencia relativa porcentual, con lo que se calculó la estimación de riesgo (odds ratio - OR) y se determinó la capacidad de cada técnica para obtener la suficiente cantidad y calidad de muestra, que permitiera obtener un diagnóstico patológico.

\section{RESULTADOS}

Se recolectaron los datos de 65 pacientes a quienes se les realizó ecoendoscopia más punción por diagnóstico de lesión sólida en páncreas, 34 (52,3\%) pacientes se realizaron con la técnica híbrida y $31(47,7 \%)$ pacientes con la TS, con estilete cuyas características se resumen en la Tabla 3. Se encontró que la frecuencia relativa porcentual respecto a la técnica de punción con aguja fina guiada por ecoendoscopia en lesiones sólidas de páncreas que permite obtener la cantidad de tejido adecuado para el diagnóstico citológico fue de $85,2 \%$ para la TH y $71 \%$ para la TS (Figura 1), por lo que se observa un aumento en el rendimiento del $14,2 \%$ a favor de la técnica húmeda híbrida, con un OR de 2,35 (IC 95\% 1,2-4,7).

\section{DISCUSIÓN}

La ultrasonografía endoscópica (USE) ofrece una excelente visualización del páncreas desde el duodeno o el estómago, lo que logra producir imágenes de alta resolución, por lo que se considera como uno de los métodos más precisos para la detección de lesiones focales pancreáticas, especialmente en pacientes con tumores pequeños ( $3 \mathrm{~cm}$ o menos) (22). Adicionalmente, la USE tiene la capacidad de obtener muestras para el diagnóstico patológico al utilizar punción guiada con aguja fina, la cual actualmente se considera una técnica de imagen segura y precisa para el diagnóstico tisular en pacientes con lesiones pancreático-biliares, siendo particularmente útil para diagnosticar tumores de páncreas y orientar la tomar decisiones terapéuticas (23). Se ha encontrado que, para estos carcinomas, esta tiene una sensibilidad diagnóstica del 54\%-96\%, una especificidad del 96\%-98\% y una precisión diagnóstica del 83\% al 95\% (24-26).

Como resultado de su utilidad demostrada se ha intentado aumentar su rendimiento diagnóstico y acercarlo cada vez más al 100\%, lo que ha impulsado el desarrollo de varias agujas para la realización de la punción, que incluyen calibres 25, 22 y 19. Particularmente, las agujas de calibre 25 son más fáciles de manejar, causan menos complicaciones (sangrado) y a su vez tienen una menor probabilidad de obtener muestras contaminadas con sangre cuando se comparan con las agujas de calibres 19 y 22 (9, 27-29). Adicionalmente, se ha demostrado que las agujas de calibre 25 tienen un mejor rendimiento diagnóstico en tumores pancreáticos sólidos, cuando se comparan con las de calibre 22 (sensibilidad combinada: 93\% para agujas de calibre 25 frente a $85 \%$ para agujas de calibre 22 para los diagnósticos realizados basados en citología) (10). 
Tabla 3. Características de los pacientes

\begin{tabular}{|c|c|c|c|c|}
\hline & $\begin{array}{l}\text { Técnica } \\
\text { húmeda }\end{array}$ & $\begin{array}{l}\text { Técnica } \\
\text { seca }\end{array}$ & $\begin{array}{c}\text { Total de } \\
\text { pacientes }\end{array}$ & $\begin{array}{l}\text { Valor } \\
\text { de } p\end{array}$ \\
\hline Número de pacientes & 34 & 31 & 65 & \\
\hline Género & & & & ns \\
\hline Masculino & 15 & 19 & 34 & \\
\hline Femenino & 19 & 12 & 31 & \\
\hline Rango de edad (años) & $29-87$ & $25-84$ & $25-87$ & \\
\hline Media & 65,3 & 63,5 & 64,4 & ns \\
\hline \multicolumn{5}{|l|}{$\begin{array}{l}\text { Diagnóstico } \\
\text { ecoendoscópico }\end{array}$} \\
\hline $\begin{array}{l}\text { Cáncer de cabeza de } \\
\text { páncreas }\end{array}$ & 26 & 23 & 49 & ns \\
\hline Cistoadenocarcinoma & 1 & & 1 & 0,32 \\
\hline $\begin{array}{l}\text { Lesión de cabeza y } \\
\text { cuerpo del páncreas }\end{array}$ & 1 & & 1 & ns \\
\hline $\begin{array}{l}\text { Lesión del cuerpo del } \\
\text { páncreas }\end{array}$ & 2 & 2 & 4 & ns \\
\hline $\begin{array}{l}\text { Lesión focal de cabeza } \\
\text { de páncreas }\end{array}$ & 2 & 3 & 5 & ns \\
\hline Pancreatitis crónica & 1 & 1 & 2 & ns \\
\hline Tumor de Franz & 1 & 1 & 2 & ns \\
\hline $\begin{array}{l}\text { Cáncer de cola de } \\
\text { páncreas }\end{array}$ & & 1 & 1 & ns \\
\hline $\begin{array}{l}\text { Tamaño de lesiones } \\
\text { (rango en milímetros) }\end{array}$ & $20-60$ & $17-50$ & $17-60$ & \\
\hline Media & 33,3 & 31,2 & 32,2 & ns \\
\hline \multicolumn{5}{|l|}{$\begin{array}{l}\text { Características } \\
\text { ecoendoscópicas }\end{array}$} \\
\hline Hipoecoica & 27 & 25 & 52 & ns \\
\hline Isoecoica & 2 & & 2 & ns \\
\hline Heterogénea & 3 & 5 & 8 & ns \\
\hline Calcificaciones & 1 & & 1 & ns \\
\hline Mixta & 1 & 1 & 2 & ns \\
\hline Rango strain ratio & $12-189$ & $13-140$ & $12-189$ & \\
\hline Media & 64,3 & 74 & 69,2 & \\
\hline \multicolumn{5}{|l|}{ Diagnóstico histopatológico } \\
\hline Adenocarcinoma & 26 & 17 & 43 & 0,0001 \\
\hline Pancreatitis crónica & 2 & 2 & 4 & ns \\
\hline Tumor de Franz & 1 & 1 & 2 & ns \\
\hline $\begin{array}{l}\text { Lesión mesenquimal } \\
\text { con atipia }\end{array}$ & & 1 & 1 & ns \\
\hline $\begin{array}{l}\text { Neoplasia papilar } \\
\text { oncótica }\end{array}$ & & 1 & 1 & ns \\
\hline No diagnóstico & 5 & 9 & 14 & 0,0001 \\
\hline
\end{tabular}

A pesar de esto, los resultados de cuatro metaanálisis disponibles sobre este tema son contradictorios. Existe una evidencia consistente de que la calidad citológica de las muestras obtenidas con las agujas 25 y 22 es similar y no se han demostrado ventajas convincentes de la aguja 25 frente a la 22, o viceversa, en términos de rendimiento técnico, facilidad de uso o perfil de seguridad. En consecuencia, en este estudio se decidió utilizar en los dos grupos, tanto para técnica húmeda como para técnica seca, agujas de calibre 22 , con el fin de evitar un factor de confusión $(8,10,11$, $18,30,31)$. Aunque esta técnica se considera como segura no está exenta de complicaciones ( $0 \%-3,4 \%)$, siendo la más frecuente la pancreatitis leve (31-33).

Adicionalmente, existen factores que aumentan el riesgo de complicaciones pospunción en relación con las características del tejido objetivo, como las masas pequeñas $(\leq 20$ $\mathrm{mm}$ ) y los tumores endocrinos (31-34). Se han informado complicaciones raras, pero graves, como sangrado $(0,2 \%)$, ruptura de seudoaneurisma, pseudoquiste pancreático, absceso y casos reportados de siembras de cáncer (31, 35-37). Las complicaciones infecciosas, bacteriemia o sepsis en las punciones de las lesiones sólidas pancreatobiliares se presentan del $0 \%-1 \%$; sin embargo, en este estudio no se presentó ninguna complicación documentada de sangrado o infección $(37,38)$.

La técnica empleada al realizar el procedimiento tiene como principio básico la visualización de la lesión diana por ultrasonografía. Se localiza el abordaje de punción (posición del transductor, presencia de vasos sanguíneos, cantidad de tejido interpuesto entre el transductor y la lesión, entre otros), se avanza una aguja elegida para puncionar la lesión, se retira el estilete (si se usa), se aplica succión, se avanza la aguja y se retira a través de la lesión para obtener material celular, finalmente, se retira la aguja y se recoge el tejido para el examen citopatológico. Distintas variaciones en la técnica han sido motivo de estudio, orientadas a determinar cuáles cambios en la técnica pueden mejorar el rendimiento diagnóstico, dentro de los que cabe resaltar la selección del sitio de punción y de la aguja, uso del estilete, succión, número de punciones y uso de un citopatólogo in situ $(23,31,39)$.

De estas modificaciones, algunas han mostrado resultados favorables respecto al aumento del rendimiento diagnóstico, dentro de las cuales se encuentran, en primer lugar, el posicionamiento; este procedimiento se realiza de una manera más cómoda cuando el ecoendoscopio se encuentra en una posición estable con la punta recta, lo que permite un fácil paso de la aguja de punción, esto generalmente se logra mejor desde la posición transgástrica que en la transduodenal $(18,40)$. Es importante recoger muestras de secciones múltiples de una lesión pancreática (múltiples punciones y técnica abanico [fanning]), ya que las lesiones neoplásicas pueden ser de naturaleza heterogénea, con cen- 


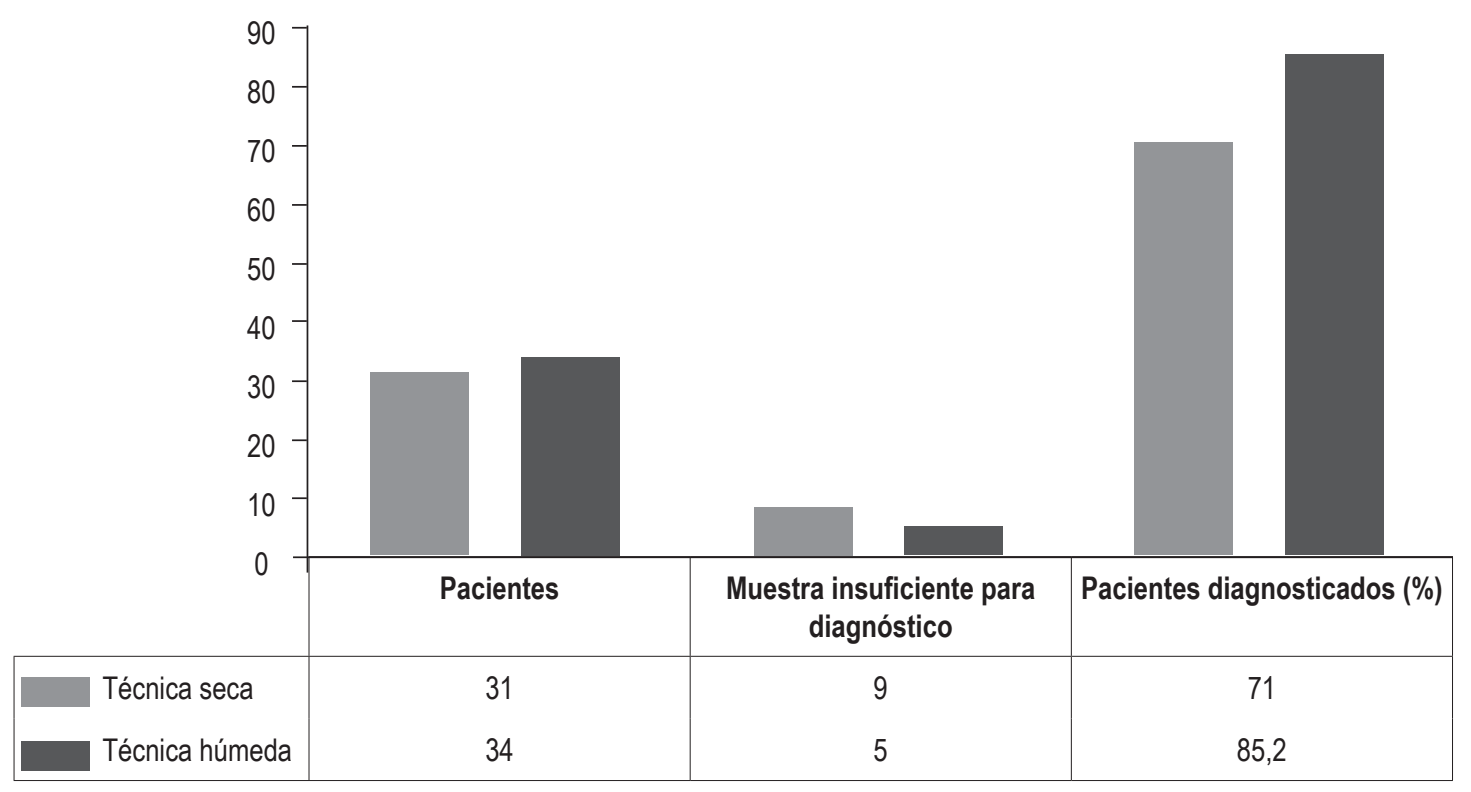

Figura 1. Comparación de técnicas

tros necróticos acelulares, por lo que es crucial enfocarse en múltiples áreas de la lesión, especialmente en la periferia. Actualmente se recomiendan 5 punciones con técnica fanning para lesiones sólidas en páncreas $(13,18,39)$. Esta técnica consiste en cambiar la posición del ángulo de la aguja utilizando los mandos y el elevador (uña) de manera intermitente para tomar muestras sucesivas de múltiples áreas de la lesión, lo que aumenta la cantidad de tejido luego de la punción, por lo que se implementó como parte del protocolo del presente estudio $(13,31,41)$.

Otro aspecto por resaltar, dada la evidencia creciente a favor, es la presencia de un citopatólogo en la sala de endoscopia donde se realiza el procedimiento denominado ROSE (por sus siglas en ingles), lo que implica la evaluación de los frotis directos obtenidos en la sala de endoscopia por un citopatólogo, que utiliza un microscopio óptico con retroalimentación inmediata al endosonografista sobre la calidad de las muestras para el diagnóstico y si se requieren muestras adicionales $(15,18,31,39,41)$.

Numerosos estudios han confirmado la superioridad de ROSE en términos de aumentar el rendimiento diagnóstico al limitar el número de pases y disminuir el número de muestras inadecuadas $(15,18,40-45)$. Sin embargo, en nuestro medio, la posibilidad de contar con un citopatólogo en la sala de endoscopia es bastante limitada, dado el aumento de los costos; por lo que en nuestro estudio no se logró adoptar esta recomendación. Se decidió realizar 5 punciones y utilizar la técnica fanning según lo recomendado por la Sociedad Europea de Endoscopia Gastrointestinal como protocolo de punción (18).
En la actualidad se sabe que la baja celularidad de los aspirados obtenidos a través de USE-PAF es una causa común de la ausencia de diagnóstico, lo que genera la necesidad de repetir los procedimientos, aumenta el costo y retrasa el diagnóstico, esto, a su vez, impide implantar estrategias de tratamiento en forma temprana, con consecuencias en la morbilidad y mortalidad para los pacientes $(44,45)$. Inicialmente se desarrollaron técnicas de succión seca y húmeda para mejorar el rendimiento de las punciones de lesiones sólidas localizadas en el mediastino o intrabdominales, pero aún no se ha recomendado como técnica USE-PAF estandarizada. Cuando se aplica la técnica seca se ha encontrado una mayor celularidad en las muestras del tejido, pero puede haber más contaminación sanguínea, por tanto, afectar la calidad general de la muestra $(20,46)$.

Respecto a la técnica húmeda, su superioridad teórica se basa en un modelo de fluido computacional tridimensional dinámico, que ha sugerido que debido a que el agua es un fluido menos compresible que el aire, una aguja llena de agua es superior a una aguja llena de aire, ya que permite una aspiración más rápida del material en el extremo distal de la aguja (17). Los resultados de nuestro estudio evidencian que la muestras obtenidas con la técnica húmeda fueron suficientes para obtener un diagnóstico patológico en el $85,2 \%$ para la técnica húmeda y el $71 \%$ para la técnica estándar, con un aumento en el rendimiento del 14,2\% respecto a la técnica húmeda. Estos resultados se correlacionan con lo encontrado por Attam y colaboradores, que compararon la técnica de succión húmeda con la técnica seca en un total 117 pacientes y mostraron que la técnica de 
succión húmeda aumentó significativamente la adquisición de tejido con mejor rendimiento diagnóstico, el cual fue calculado de $85,5 \%$ versus $75,2 \%(P<0,035)$; sin embargo, no hubo diferencia en la cantidad de contaminación sanguínea entre las 2 técnicas (19).

Otro estudio piloto realizado por Berzosa y colaboradores en 15 pacientes con lesiones sólidas, en el que se comparó las técnicas de aspiración húmeda, híbrida y seca de USE-PAF, en donde el objetivo fue determinar la muestra adecuada para proporcionar un diagnóstico patológico final, así como el volumen de material aspirado y el rendimiento diagnóstico (maligno o no maligno) para cada técnica (47). No se encontraron diferencias significativas entre las técnicas híbridas, húmedas y secas $(87 \%, 87 \%$ y $67 \%$, respectivamente), sin embargo, esta diferencia tal vez no fue detectada en este estudio piloto dada su poca potencia estadística (17).

Aunque aún no se conoce la causa exacta por la que la técnica húmeda proporciona una mayor celularidad en las muestras obtenidas, existen diferentes teorías, algunas de ellas basadas en modelos computacionales que muestran que una aguja llena de agua es superior a una aguja llena de aire, ya que permite una aspiración más rápida del material en el extremo distal de la aguja, como ya habíamos anotado, lo que permite una mejor transmisión de la succión aplicada, en comparación con una columna de aire dentro de la aguja. La solución salina puede recubrir el revestimiento interno de la aguja, por tanto, cambiar las propiedades de la superficie, lo que facilita el movimiento del aspirado hacia la aguja. Además, la columna de solución salina puede actuar como un estilete, lo que reduce potencialmente la contaminación del tejido durante la punción en una lesión y mantiene la aguja desatascada $(17,18,47)$.

Adicionalmente, la técnica húmeda permite un cambio en las propiedades de la superficie interna de la aguja hueca cuando está recubierta con solución salina normal, lo que puede reducir la fricción entre el aspirado de tejido y la pared de la aguja, esto permite un movimiento más suave del material dentro del canal de la aguja (17). Dadas las condiciones en las que se realizó este estudio, consideramos que la principal limitante es la ausencia de la evaluación rápida en la sala de endoscopia por un citopatólogo, sin embargo, consideramos que en la mayoría de los centros de endoscopia en Colombia no es factible, esto, como ya fue anotado, puede conducir a un aumento en el número de muestras inadecuadas, por tanto, puede afectar el rendimiento diagnóstico (48). Otra limitante es que el tamaño de la muestra es pequeño, aunque este es mucho mayor que el estudio de Barsa y colaboradores. También, sería importante que se hubiera medido el volumen de material en cada grupo, sin embargo, nuestro objetivo era la sensibilidad en el diagnóstico.

\section{CONCLUSIÓN}

Los hallazgos previamente descritos sugieren que la técnica húmeda híbrida aumenta la celularidad significativamente en las muestras obtenidas de lesiones sólidas del páncreas cuando se compara con la técnica convencional, siendo una técnica de fácil aplicación en el contexto de la ausencia de un citopatólogo en la sala de endoscopia. Además, la implementación de esta técnica no implica un costo adicional y, dado que este trabajo y otro internacional (más pequeño) sugieren esta superioridad, debería ser la técnica de elección cuando se punciona una lesión sólida del páncreas.

\section{REFERENCIAS}

1. Low G, Panu A, Millo N, Leen E. Multimodality imaging of neoplastic and nonneoplastic solid lesions of the pancreas. Radiographics. 2011;31:993-1015. doi: https://doi. org/10.1148/rg.314105731.

2. Ferlay J, Soerjomataram I, Dikshit R, Eser S, Mathers C, Rebelo $\mathrm{M}$, et al. Cancer incidence and mortality worldwide: sources, methods and major patterns in GLOBOCAN 2012. Int J Cancer. 2015;136:E359-86. doi: https://doi. org/10.1002/ijc.29210.

3. Liles J, Katz M. Pancreaticoduodenectomy with vascular resection for pancreatic head adenocarcinoma. Expert Rev Anticancer Ther. 2014;14:919-29. doi: https://doi.org/10. 1586/14737140.2014.919860.

4. Scialpi M, Reginelli A, D’Andrea A, Gravante S, Falcone G, Baccari $P$, et al. Pancreatic tumors imaging: an update. Int J Surg. 2016;(1):S142-55. doi: https://doi.org/10.1016/j. ijsu.2015.12.053.

5. Wang W, Shpaner A, Krishna S, Ross W, Bhutani M, Tamm EP, et al. Use of EUS-FNA in diagnosing pancreatic neoplasm without a definitive mas son CT. Gastrointest Endosc. 2013;78:73-80. doi: https://doi.org/10.1016/j. gie.2013.01.040.

6. Hewitt M, McPhail M, Possamai L, Dhar A, Vlavianos P, Monahan K. EUS-guided FNA for diagnosis of solid pancreatic neoplasms: a meta-analysis. Gastrointest Endosc. 2012;75:31931. doi: https://doi.org/10.1016/j.gie.2011.08.049.

7. Eloubeidi M, Tamhane A. EUS-guided FNA of solid pancreatic masses: a learning curve with 300 consecutive procedures. Gastrointest Endosc. 2005;61:700-8. doi: https:// doi.org/10.1016/S0016-5107(05)00363-9.

8. Korenblit J, Tholey D, Tolin J, Loren D, Kowalski T, Adler DG, et al. Effect of the time of day and queue position in the endoscopic schedule on the performance characteristics of endoscopic ultrasound-guided fine-needle aspiration for diagnosing pancreatic malignancies. Endosc Ultrasound. 2016;5:78-84. doi: https://doi.org/10.4103/2303-9027.180470.

9. Ramesh J, Bang J, Hebert-Magee S, Trevino J, Eltoum I, Frost A, et al. Randomized trial comparing the flexible 19G and 25G needle for endoscopic ultrasound-guided fine needle aspiration of solid pancreatic mass lesions. 
Pancreas. 2015;44:128-33. doi: https://doi.org/10.1097/ MPA.0000000000000217.

10. Madhoun M, Wani S, Rastogi A, Early D, Gaddam S, Tierney $\mathrm{W}$, et al. The diagnostic accuracy of 22-gauge and 25-gauge needles in endoscopic ultrasound-guided fine needle aspiration of solid pancreatic lesions: a metaanalysis. Endoscopy. 2013;45:86-92. doi: https://doi. org/10.1055/s-0032-1325992.

11. Kamata K, Kitano M, Yasukawa S, Kudo M, Chiba Y, Ogura $\mathrm{T}$, et al. Histologic diagnosis of pancreatic masses using 25-gauge endoscopic ultrasound needles with and without core trap: a multicenter randomized trial. Endoscopy. 2016;48:632-8. doi: https://doi. org/10.1055/s-0042-106294.

12. Nakai $Y$, Isayama $H$, Chang $K$, Yamamoto N, Hamada T, Uchino $\mathrm{R}$, et al. Slow pull versus suction in endoscopic ultrasound-guided fine-needle aspiration of pancreatic solid masses. Dig Dis Sci. 2014;59:1578-85. doi: https://doi. org/10.1007/s10620-013-3019-9.

13. Bang J, Magee S, Ramesh J, Trevino J, Varadarajulu S. Randomized trial comparing fanning with standard technique for endoscopic ultrasound-guided fine-needle aspiration of solid pancreatic mass lesions. Endoscopy. 2013;45:44550. doi: https://doi.org/10.1055/s-0032-1326268.

14. Suzuki R, Irisawa A, Bhutani M, Hikichi T, Takagi T, Sato A, et al. Prospective evaluation of the optimal number of 25-gauge needle passes for endoscopic ultrasound-guided fine-needle aspiration biopsy of solid pancreatic lesions in the absence of an onsite cytopathologist. Dig Endosc. 2012;24:452-6. doi: https://doi.org/10.1111/j.14431661.2012.01311.x.

15. Hebert-Magee S, Bae S, Varadarajulu S, Ramesh J, Frost $\mathrm{R}$, Eloubeidi MA, et al. The presence of a cytopathologist increases the diagnostic accuracy of endoscopic ultrasoundguided fine needle aspiration cytology for pancreatic adenocarcinoma: a meta-analysis. Cytopathology. 2013;24:15971. doi: https://doi.org/10.1111/cyt.12071.

16. Varadarajulu S, Tamhane A, Eloubeidi M. Yield ofEUS-guided FNA of pancreatic masses in the presence or the absence of chronic pancreatitis. Gastrointest Endosc. 2005;62:728-36. doi: https://doi.org/10.1016/j.gie.2005.06.051.

17. Villa N, Berzosa M, Wallace M, Raijman I. Endoscopic ultrasound-guided fine needle aspiration: the wet suction technique. Endosc Ultrasound. 2016;5:17-20. doi: https:// doi.org/10.4103/2303-9027.175877.

18. Polkowski M, Jenssen C, Kaye P, Carrara S, Deprez P, Gines A, et al. Technical aspects of endoscopic ultrasound (EUS)guided sampling in gastroenterology: European Society of Gastrointestinal Endoscopy (ESGE) Technical Guideline March 2017. Endoscopy. 2017;49:989-1006. doi: https:// doi.org/10.1055/s-0043-119219.

19. Attam R, Arain M, Bloechl S, Trikudanathan G, Munigala S, Bakman Y, et al. "Wet suction technique (WEST)": a novel way to enhance the quality of EUS-FNA aspirate. Results of a prospective, single-blind, randomized, controlled trial using a 22-gauge needle for EUS-FNA of solids lesions.
Gastrointest Endosc. 2015;81:1401-7. doi: https://doi. org/10.1016/j.gie.2014.11.023.

20. Lee J, Choi J, Lee K, Kim K, Shin J, Lee JK, et al. A prospective, comparative trial to optimize sampling techniques in EUS- guided FNA of solid pancreatic masses. Gastrointest Endosc. 2013;77:745-51. doi: https://doi.org/10.1016/j. gie.2012.12.009.

21. Skelton W, Parekh H, Starr J, Trevino J, Cioffi J, Hughes S, et al. Clinical factors as a component of the personalized treatment approach to advanced pancreatic cancer: a systematic literature review. J Gastrointest Cancer. 2018;49(1):18. doi: 10.1007/s12029-017-0021-z.

22. Lee E, Lee J. Imaging diagnosis of pancreatic cancer: a stateof-the-art review. World J Gastroenterol. 2014;20:7864-77. doi: https://doi.org/10.3748/wjg.v20.i24.7864.

23. Han J, Chang K. Endoscopic ultrasound-guided direct intervention for solid pancreatic tumors. Clin Endosc. 2017;50:126-37. doi: https://doi.org/10.5946/ce.2017.034.

24. Chen G, Liu S, Zhao Y, Dai M, Zhang T. Diagnostic accuracy of endoscopic ultrasound-guided ne-needle aspiration for pancreatic cancer: a meta-analysis. Pancreatology. 2013;13:298304. doi: https://doi.org/10.1016/j.pan.2013.01.013.

25. Puli S, Bechtold M, Buxbaum J, Eloubeidi M. How good is endoscopic ultrasound-guided ne-needle aspiration in diagnosing the correct etiology for a solid pancreatic mass?: a meta-analysis and systematic review. Pancreas. 2013;42:206. doi: https://doi.org/10.1097/MPA.0b013e3182546e79.

26. Banafea O, Mghanga F, Zhao J, Zhao R, Zhu L. Endoscopic ultrasonography with fine-needle aspiration for histological diagnosis of solid pancreatic masses: a meta-analysis of diagnostic accuracy studies. BMC Gastroenterol. 2016;16:108. doi: https://doi.org/10.1186/s12876-016-0519-z.

27. Varadarajulu S, Bang J, Holt B, Hasan M, Logue A, Hawes R, et al. The 25-gauge EUS-FNA needle: Good for on-site but poor for off-site evaluation? Results of a randomized trial. Gastrointest Endosc. 2014;80:1056-63. doi: https://doi. org/10.1016/j.gie.2014.05.304.

28. Rastogi A, Wani S, Gupta N, Singh V, Gaddam S, Reddymasu $\mathrm{S}$, et al. A prospective, single-blind, randomized, controlled trial of EUS-guided FNA with and without a stylet. Gastrointest Endosc. 2011;74:58-64. doi: https://doi. org/10.1016/j.gie.2011.02.015

29. Kamata K, Kitano M, Omoto S, Kadosaka K, Miyata T, Minaga $\mathrm{K}$, et al. New endoscopic ultrasonography techniques for pancreaticobiliary diseases. Ultrasonography. 2016;35:169-79. doi: https://doi.org/10.14366/usg.15042.

30. Larghi A, Iglesias-García J, Poley J, Monges G, Petrone M, Rindi G, et al. Feasibility and yield of a novel 22-gauge histology EUS needle in patients with pancreatic masses: a multicenter prospective cohort study. Surg Endosc. 2013;27:37338. doi: https://doi.org/10.1007/s00464-013-2957-9.

31. Matsubayashi H, Matsui T, Yabuuchi Y, Imai K, Tanaka M, Kakushima N, et al. Endoscopic ultrasonography guidedfine needle aspiration for the diagnosis of solid pancreaticobiliary lesions: Clinical aspects to improve the diagnosis. 
World J Gastroenterol. 2016;22:628-40. doi: https://doi. org/10.3748/wjg.v22.i2.628.

32. Yasuda I, Iwashita T, Doi S. Tips for endoscopic ultrasoundguided fine needle aspiration of various pancreatic lesions. J Hepatobiliary Pancreat Sci. 2014;21:E29-33. doi: https:// doi.org/10.1002/jhbp.60.

33. Haba S, Yamao K, Bhatia V, Mizuno N, Hara K, Hijioka S, et al. Diagnostic ability and factors affecting accuracy of endoscopic ultrasound-guided fine needle aspiration for pancreatic solid lesions: Japanese large single center experience. J Gastroenterol. 2013;48:973-81. doi: https://doi. org/10.1007/s00535-012-0695-8.

34. Puri R, Vilmann P, Săftoiu A, Skov B, Linnemann D, Hassan $\mathrm{H}$, et al. Randomized controlled trial of endoscopic ultrasound-guided fine-needle sampling with or without suction for better cytological diagnosis. Scand J Gastroenterol. 2009;44:499-504. doi: https://doi. org/10.1080/00365520802647392.

35. Hamada T, Yasunaga $H$, Nakai $Y$, Isayama $H$, Horiguchi $H$, Matsuda $S$, et al. Severe bleeding and perforation are rare complications of endoscopic ultrasound-guided fine needle aspiration for pancreatic masses: an analysis of 3,090 patients from 212 hospitals. Gut Liver. 2014;8:215-8. doi: https://doi.org/10.5009/gnl.2014.8.2.215.

36. Wang J, Zhao S, Chen Y, Jia R, Zhang X. Endoscopic ultrasound guided fine needle aspiration versus endoscopic ultrasound guided fine needle biopsy in sampling pancreatic masses: a meta-analysis. Medicine (Baltimore). 2017;96:e7452. doi: https://doi.org/10.1097/MD.0000000000007452.

37. Katanuma A, Maguchi $H$, Hashigo S, Kaneko M, Kin T, Yane K, et al. Tumor seeding after endoscopic ultrasoundguided fine-needle aspiration of cancer in the body of the pancreas. Endoscopy. 2012;44:E160-1. doi: https://doi. org/10.1055/s-0031-1291716.

38. Fisher L, Segarajasingam D, Stewart C, Deboer W, Yusoff I. Endoscopic ultrasound guided fine needle aspiration of solid pancreatic lesions: Performance and outcomes. J Gastroenterol Hepatol. 2009;24:90-6. doi: https://doi. org/10.1111/j.1440-1746.2008.05569.x.

39. Storm A, Lee L. Endoscopic ultrasound-guided techniques for diagnosing pancreatic mass lesions: Can we do better? World J Gastroenterol. 2016;22:8658-69. doi: https://doi. org/10.3748/wjg.v22.i39.8658.
40. Artifon E, Guedes H, Cheng S. Maximizing the diagnostic yield of endoscopic ultrasound-guided fine-needle aspiration biopsy. Gastroenterology. 2017;153:881-5. doi: https://doi.org/10.1053/j.gastro.2017.08.058.

41. Holt B, Varadarajulu S, Hébert-Magee S. High-quality endoscopic ultrasound-guided fine needle aspiration tissue acquisition. Adv Ther. 2014;31:696-707. doi: https://doi. org/10.1007/s12325-014-0129-5.

42. Bhatia V, Varadarajulu S. Endoscopic ultrasonographyguided tissue acquisition: how to achieve excellence. Dig Endosc. 2017;29:417-30. doi: https://doi.org/10.1111/ den.12823.

43. Yamabe A, Irisawa A, Bhutani M, Shibukawa G, Fujisawa $\mathrm{M}$, Sato A, et al. Efforts to improve the diagnostic accuracy of endoscopic ultrasound-guided fine-needle aspiration for pancreatic tumors. Endosc Ultrasound. 2016;5:225-32. doi: https://doi.org/10.4103/2303-9027.187862.

44. Wani S. Basic techniques in endoscopic ultrasound-guided fine-needle aspiration: role of a stylet and suction. Endosc Ultrasound. 2014;3:17-21. doi: https://doi. org/10.4103/2303-9027.123008.

45. Costache M, Iordache S, Karstensen J, Săftoiu A, Vilmann P. Endoscopic ultrasound-guided fine needle aspiration: from the past to the future. Endosc Ultrasound. 2013;2:77-85. doi: https://doi.org/10.4103/2303-9027.117691.

46. Wang Y, Chen $\mathrm{Q}$, Wang J, Wu X, Duan Y, Yin P, et al. Comparison of modified wet suction technique and dry suction technique in endoscopic ultrasound-guided fineneedle aspiration (EUS-FNA) for solid lesions: study protocol for a randomized controlled trial. Trials. 2018;19:45. doi: https://doi.org/10.1186/s13063-017-2380-y.

47. Berzosa M, Villa N, Bartel M, Wallace M, Tau J, Trang T, et al. Pilot study comparing hybrid vs. wet vs. dry suction techniques for EUS-FNA of solid lesions. Gastrointest Endos. 2014;79:AB430. doi: https://doi.org/10.1016/j. gie.2014.02.597.

48. Iwashita T, Yasuda I, Mukai T, Doi S, Nakashima M, Uemura $S$, et al. Macroscopic on-site quality evaluation of biopsy specimens to improve the diagnostic accuracy during EUSguided FNA using a 19-gauge needle for solid lesions: a single-center prospective pilot study (MOSE study) Gastrointest Endosc. 2015;81:177-85. doi: https://doi. org/10.1016/j.gie.2014.08.040. 